\title{
Cyclic feedback systems with quorum sensing coupling
}

\section{Authors: Tomáš Gedeon, Mark Pernarowski, and Adam Wilander}

The final publication is available at Springer via http://dx.doi.org/10.1007/s11538-016-0187-8.

Gedeon, Tomas, Mark Pernarowski, and Adam Wilander. "Cyclic Feedback Systems with Quorum Sensing Coupling." Bulletin of Mathematical Biology.

Made available through Montana State University's $\underline{\text { ScholarWorks }}$

scholarworks. montana.edu 


\title{
Cyclic feedback systems with quorum sensing coupling.
}

\author{
Tomas Gedeon, Mark Pernarowski, Adam Wilander * \\ Department of Mathematical Sciences \\ Montana State University, MT, 59715, USA \\ gedeon@math.montana.edu
}

May 31, 2016

\begin{abstract}
Synchronization and desynchronzation is of great interest in the study of circadian rhythms, metabolic oscillations and time dependent cell aggregate behaviors. Several recent studies examine synchronization and other dynamics in models of repressillators coupled by a quorum sensing mechanism that uses a diffusive signal. Their numerical simulations have shown the complexity of the collective behavior depends sensitively on which protein upregulates diffusive signal.

In this paper we rigorously prove that the collective dynamics indeed strongly depends on how the signaling network integrates into the repressillator network. In fact we prove a general result for a class of negative cyclic feedback systems with signaling of which the repressilator is but one example. We show that if the feedback along the signaling loop is also negative, the resulting negative feedback, negative signaling (Nf-Ns) system admits either unique stable equilibrium, or a stable oscillation. When a positive signaling feedback is included the system is no longer (Nf-Ns) and numerically exhibits multistable dynamics (as in $[25,26])$. We demonstrate that this multistability emerges through saddle node bifurcations of a sole cubic curve - as in generic bistable models.
\end{abstract}

\section{Introduction}

Cellular populations need to communicate to facilitate joint, synchronous responses to outside stimuli or to produce a sustained rhythmical output. There has been strong recent interest in mechanisms by which such populations achieve and maintain synchrony. Typically intracellular proteins promote the production of signaling molecules. These diffuse in the extracellular spaces to provide a communication mechanism for the cellular aggregate. When cells use the signaling molecule to sense density of the cell population and use this information to

\footnotetext{
${ }^{*}$ we also wish to acknowledge Shuai Zhao for his efforts on this project at its inception.
} 
up-regulate their gene expression, such communication is referred to as quorum sensing, and the signaling molecule is typically referred to as an autoinducer. Many such quorum sensing mechanisms have been discovered and modeled. Of particular interest is the emergence and loss of synchronized behavior when the cell density, or some other relevant parameter that characterizes the size of the population is changed.

De Monte et. al. [3] studied glycolitic oscillations in yeast both experimentally and theoretically. They observed the loss of population wide oscillations as the density of the cell decreased. They found that the loss of synchronous oscillations was not the result of de-synchronization of individual oscillators and that below a certain density threshold all cells stopped oscillating and entered a steady state. They called this transition dynamic quorum sensing.

Taylor et. al. [24] studied transition to synchronization in a large population of chemical oscillators (modeled by Belousov-Zhabotniskii equations) as a function of population density in low stirring rate and high stirring rate regimes. These correspond to low and high transport rates of the signaling species, respectively. They found that the transition to synchrony is different in these two cases. While at a high stirring rate, the transition is gradual and oscillators are recruited to the synchronized pool gradually. Conversely, at a low stirring rate the emergence of synchrony is sudden at a certain density.

In this paper we study the emergence of oscillations and complex dynamics in variants and generalizations of a synthetic biology model first introduced by Garcia-Ojalvo et. al. [16]. Their model consists of a repressilator coupled to a quorum sensing module from Vibrio fischeri. Each cell in the aggregate is equipped with a three gene network that admits autonomous oscillations and is connected to a Vibrio fischeri module that produces and responds to a diffusible autoinducer AI. The transmembrane transport of the autoinducer is assumed proportional to the difference between the aggregate average and the local concentration. In their model only Tet $R$ promotes the autoinducer. This feedback can produce stable steady behavior, and both synchronous and asynchronous oscillations [18]. However, when a different network protein promotes the autoinducer, much more complicated dynamics can occur. Ulner et. al. [26] and Koseska et. al. [11] examined the dynamics of small cell aggregates when $C I$ promoted the autoinducer. Such systems exhibit complex multistable behavior. Although synchronous steady and oscillatory states are also possible, much of the aggregate behavior is a mixture steady and oscillatory states. All of these behaviors depend greatly on parameter values and the number of cells $N$.

Here we present a unified approach for categorizing and analyzing all such models. In the aforementioned studies, cells had a $K=3$ gene network, and one protein $\Omega$ promoted the autoinducer $S$. We illustrate three such networks in Figure 1 . When $\Omega=A$ the dynamics are simple in the sense that the $N=1$ system has a unique equilibrium (Figure 1(a)). For $N \geq 2$ we show that two Hopf branches emerge, one of which is dependent on the quorum sensing parameter $q$. Since such systems have a unique equilibria, we can show the aggregate remains (locally) monostable between synchronous equilibria and synchronous periodic orbits. We prove the majority of these results for a much larger class of Nf-Ns (Negative feedback 
- Negative sensing) models having $n$ genes. Such models are defined in Section 2 and have a unique synchronous equilibria which can destabilize only by a Hopf bifurcation. Lastly, we numerically demonstrate that for one such model only the quorum or $q$-dependent Hopf branch $\left(H B_{1}\right)$ has emergent stable period orbits (cf. Figure 4,5).

(a)

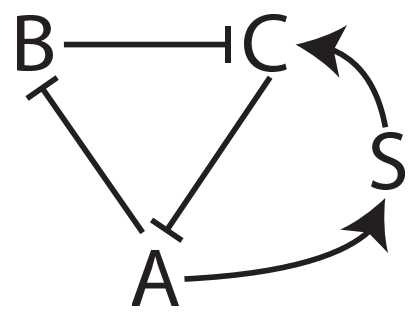

MONOSTABLE (b)

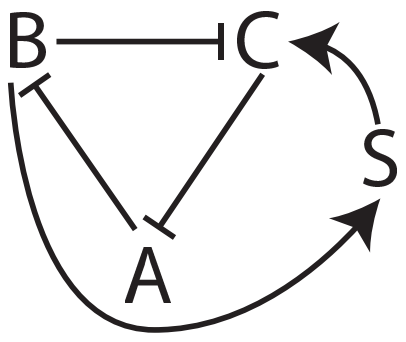

BISTABLE (c)

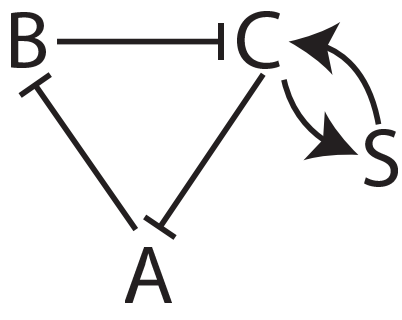

BISTABLE

Figure 1: Schematics for three different $K=3$ repressillator circuits. In each circuit $A=$ Tet $R, B=C I, C=L a c I$ and a different protein $\Omega \in\{A, B, C\}$ upregulates the diffusive signal $S$. This autoinducer $S$ upregulates $C$ in all networks. When $\Omega=A$ the network dynamics are monostable (simple). Otherwise, the network dynamics can be bistable (complex).

Aggregate dynamics are very different when other proteins $\Omega$ promote the autoinducer in the repressilator model. We show that, when $\Omega=C I$ or LacI (Figure 1(b)-(c) with $\Omega=B, C$ ) the existence of equilibria in the aggregate depends only on knowing the roots of a single scalar function whose root locus is cubic shaped. Such is the case in many bistable systems. In this case we show the saddle node bifurcations define parameter sets where the aggregate is either monostable or has a huge numbers of equilibria. Koseska et. al. [11] numerically demonstrated ( $N=5$ cells) how these equilibria also lead to numerous new Hopf bifurcations and hence prolific multistability.

In Section 2, we define both the Garcia-Ojalvo et. al. [16] and Nf-Ns models. The latter model generalizes the former but can have an arbitrary number of genes $n$. Both are shown to have a Jacobian with a special block structure. In Section 3, we exploit this block structure to show the stability of synchronous equilibria depends only on one $(2 n+1)^{t h}$ degree polynomial where $n$ is the number of genes in the model. Noteably, the stability is independent of the number of cells $N$. In Section 4, we prove the Nf-Ns system has a sole (synchronous) equilibria that can only destabilize via a Hopf bifurcation. Since the Garcia-Ojalvo et. al. [16] model is an 
Nf-Ns system the same results hold. For the former model we additionally show only two Hopf branches exist and only the quorum sensing dependent one is stable. In Section 5 we then show that for the $\Omega=B, C^{1}$ feedback cases the system may have multiple equilibria. In this bistable case, a systematic description of all such equilibria is given in terms of a "consistency" condition.

\section{Cyclic Feedback Models with Quorum Sensing}

Most recent repressillator models incorporating quorum sensing [3, 11, 18, 25, 26, 27] are variations or simplifications of the model due to Garcia-Ojalvo et al. [16]. In all these studies, either TetR (A) or $C I$ (B) promotes the production of the autoinducer involved the quorum sensing. When Tet $R$ promotes the autoinducer, the resulting dynamics are relatively simple (monostable case). However, when $C I$ promotes the autoinducer, the dynamics can be very complex (bistable case). We cast all models into a general construct more amenable for local analyses and helpful for making simple generalizations regarding network dynamics.

We first define the model of Garcia-Ojalvo, Ellowitz and Strogatz [16] who studied a synthetic gene network which included quorum sensing for synchronization. A subsequent numerical bifurcation study can be found in Potapov et al. [18]. The model network illustrated in Figure 2 has two basic parts. One is a sub-network of three genes that repress each other in a cycle. Each gene is modeled by two variables, one representing abundance of mRNA and the other abundance of the corresponding protein. Specifically, the gene lacI expresses its protein LacI, and LacI inhibits transcription of the gene tetR. The protein TetR expressed by the gene tetR inhibits transcription of the gene cI. Lastly, protein CI, expressed by the gene cI, inhibits transcription of the gene lacI, thus completing the cycle. Every protein competes with polymerase for binding sites on a promoter and in this way inhibits the transcription from DNA to the corresponding mRNA. Therefore, as more protein is produced, less mRNA of the subsequent gene is produced. The other part of the network is another gene, luxI, upregulated by tetR protein, produces protein LuxI which synthesizes a small molecule known as an autoinducer AI. This autoinducer (acylated homoserine lactone) can diffuse freely through the cell membrane and the extracellular medium. The combination of AI and a second protein (LuxR) will activate transcription of the gene lacI. Therefore, the mRNA (lacI) dynamics are different from the other two mRNA. Under these and other assumptions the equations describing the protein and mRNA concentrations in the $i^{\text {th }}$ cell in a collection of $N$ cells are:

\footnotetext{
${ }^{1}$ or CI and LacI, respectively
} 


$$
\begin{array}{rrrl}
\dot{a}_{i} & = & -a_{i}+\Phi\left(C_{i}\right) & (\text { tet }) \\
\dot{A}_{i} & & -\beta A_{i}+\beta a_{i} & (\text { Tet }) \\
\dot{b}_{i} & -b_{i}+\Phi\left(A_{i}\right) & (\text { cI }) \\
\dot{B}_{i} & -\beta B_{i}+\beta b_{i} & (\text { CI }) \\
\dot{c}_{i} & -c_{i}+\Phi\left(B_{i}\right)+\Gamma\left(S_{i}\right) & (\text { lacI }) \\
\dot{C}_{i} & -\beta C_{i}+\beta c_{i} & (\text { LacI }) \\
\dot{S}_{i}= & -\left(k_{s 0}+\eta\right) S_{i}+k_{s 1} A_{i}+\frac{\eta q}{N} \sum_{j=1}^{N} S_{j} & (\text { AI })
\end{array}
$$

where

$$
\begin{aligned}
\Phi(z) & =\frac{\alpha}{1+z^{n}} \\
\Gamma(z) & =\frac{\kappa z^{m}}{1+z^{m}}
\end{aligned}
$$

are the assumed nonlinearities for transcription feedback by the proteins and autoinducer. The system (2.1) models the network in Figure 1(a). Inhibitory feedback within the network occurs through the bounded and decreasing function $\Phi(z)$. Conversely, the positive autoinducer feedback occurs through $\Gamma(z)$ which is bounded and increasing. Below we generalize the model above in several ways. Most notably, some monotonicity properties on $\Phi(z)$ are relaxed resulting in what will later be defined as the Nf-Ns model. But for numerical calculations we use (2.1) with $\Phi(z)$ and $\Gamma(z)$ defined above as are used in preceding studies $[16,11,18,25,26]$.

A lowercase convention is used to indicate mRNA concentrations whereas uppercase indicates their associated protein. Thus, $a_{i}$ is the (tetR) mRNA concentration in the $i$-th cell and $A_{i}$ is the associated (TetR) protein concentration. The (all to all) coupling between cells is evident in the last term of $\dot{S}_{i}$. In (2.1) the parameter $\eta$ is the permeability of the cell membrane to the autoinducer $S_{i}$. The quorum sensing parameter $q \in(0,1)$ is derived from a quasi-steady state approximation to the equation for extracellular autoinducer concentration $S_{e}$ :

$$
\frac{d S_{e}}{d t}=-k_{s e} S_{e}+k_{\mathrm{diff}}\left(\bar{S}-S_{e}\right) \quad, \quad \bar{S}=\frac{1}{N} \sum_{i=1}^{N} S_{i}
$$

Such an approximation yields

$$
q=\frac{k_{\mathrm{diff}}}{k_{s e}+k_{\mathrm{diff}}}=\frac{\rho_{e}}{k_{s e}+\rho_{e}}
$$

which is roughly proportional to cell density $\rho_{e}$ when $\rho_{e} \ll k_{s e}[16]$. Thus, at low cell densities, $q$ is small while at large densities $q$ is near unity. 


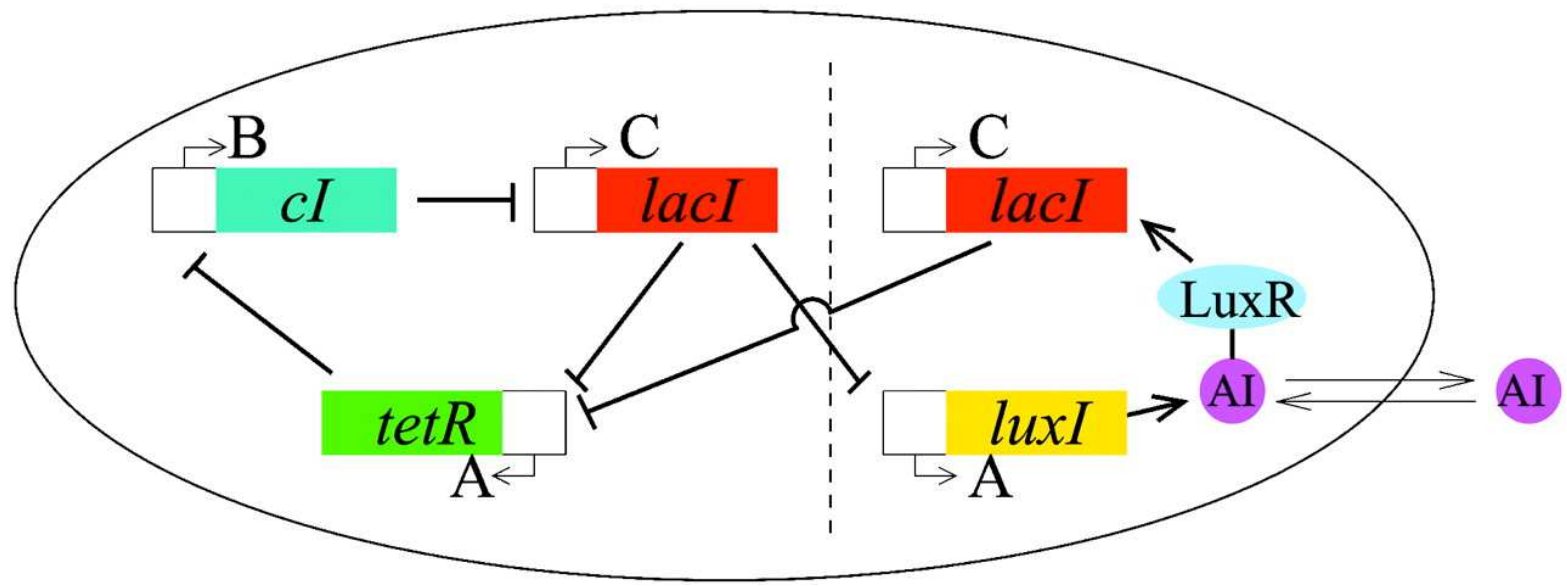

Figure 2: A synthetic gene network in E. Coli proposed by Garcia-Ojalvo J et al. [16]. The left portion of the network is the standard repressilator model. The right side includes a TetR mechanism for autoinducer (AI) production. The autoinducer passively diffuses into extracelluar regions which enables quorum sensing in a cell collective.

To completely define our more general Nf-Ns model, we first generalize the intracellular network of Garcia-Ojalvo et. al [16]. Instead of a network of $K=3$ genes we consider a general cycle of $K=n$ genes in cell $i$. We continue to represent each gene $j$ by two variables $X_{j}$ (representing mRNA concentration) and $x_{j}$ (representing protein concentration)

$$
\begin{aligned}
\dot{x}_{j} & =-c_{j} x_{j}+\Phi_{j}\left(X_{j-1}\right) \\
\dot{X}_{j} & =b_{j} x_{j}-a_{j} X_{j} \quad, \quad j=1, \ldots, n-1
\end{aligned}
$$

The indices are considered modulo $n$ and so $x_{0}$ is identified with $x_{n}$. Without loss of generality we assume that the feedback from the autoinducer affects variable $x_{n}$

$$
\begin{aligned}
\dot{x}_{n} & =-c_{n} x_{n}+\Phi_{n}\left(X_{n-1}\right)+\Gamma\left(S_{i}\right), \\
\dot{X}_{n} & =b_{n} x_{n}-a_{n} X_{n} .
\end{aligned}
$$

In (2.2)-(2.3), all the constants $a_{j}, b_{j}, c_{j}$ are assumed to be positive and $\Gamma\left(S_{i}\right)$ is any positive, bounded and increasing function of the autoinducer concentration $S_{i}$ in cell $i$. The functions $\Phi_{j}(z)$ need not be the same but must be positive, bounded and monotonic. In particular, $\Phi_{j}(z)$ may be either increasing or decreasing. This should be contrasted to the model (2.1) where $\Phi_{j}(z)=\Phi(z)$ for all $j=1,2,3$ and all $\Phi_{j}(z)$ were decreasing.

We examine the effect of differing autoinducer feedback mechanisms on the global dynamics of the quorum. Specifically, we examine the system above, where the synchronizing signal is placed at a different position in the loop.

$$
\dot{S}_{i}=-\left(k_{s 0}+\eta\right) S_{i}+k_{s 1} \Omega+\frac{\eta q}{N} \sum_{i=1}^{N} S_{i} \quad, \quad \Omega \in\left\{X_{1}, X_{2}, \ldots, X_{n}\right\}
$$


As depicted in Figure 3 there are two feedback loops in the system (2.2)-(2.4). The first, internal feedback loop consists of $X_{1}, \ldots, X_{n}$ and interactions described in (2.2) and (2.3). The second, quorum sensing feedback loop consists of $X_{1}, X_{2}, \ldots, X_{k}=\Omega, S_{i}, X_{n}$. The quorum sensing loop couples the cells in the system together. We now associate a sign to each loop.

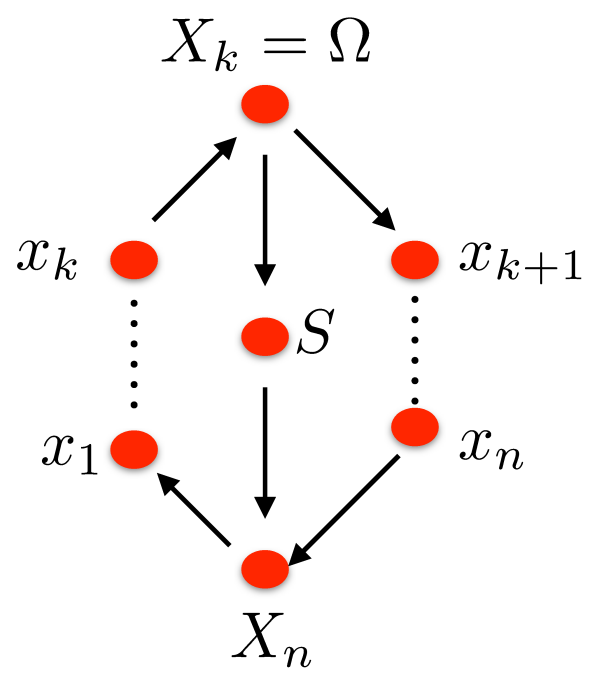

Figure 3: A schematic of the Nf-Ns system which generalizes the standard represillator model. The system has $n$ genes and therefore $2 n$ nodes along the perimeter cycle, see (2.2)-(2.4). The synchronizing signal $\Omega=X_{k}$ can occur at different positions in the perimeter cycles. We require that the number of negative feedbacks along the perimeter cycle is odd $(\bar{\beta}=-1)$ and also that the number of negative feedbacks along the left loop that includes $\Omega \rightarrow S \rightarrow x_{n}$ is odd $(\bar{\alpha}=-1)$.

Definition 2.1 Let $\delta_{i}=\operatorname{sgn} \Phi_{i}^{\prime}$. Let

$$
\bar{\alpha}:=\Pi_{i=1}^{n} \delta_{i}, \quad \bar{\beta}:=\Pi_{i=1}^{k} \delta_{i}, \text { where } k \text { is the index such that } X_{k}=\Omega \text {. }
$$

We say that the internal loop has a negative (positive) feedback if $\bar{\alpha}=-1(\bar{\alpha}=1)$ respectively. We say that the coupling loop has a negative (positive) feedback if $\bar{\beta}=-1(\bar{\beta}=1)$ respectively.

Note that this definition of negative and positive feedback agrees with the standard definition for cyclic feedback systems $[13,8,9]$. Theory developed in these papers shows that negative 
feedback systems with sufficiently high gain $[23,22]$ admit a stable periodic orbit, while positive feedback systems do not. Since we are interested in synchronization of stable oscillations, we will restrict ourselves to $\bar{\alpha}=-1$ i.e. the case when the internal loop is negative. Clearly, the repressilator studied in [16] falls into this category.

Definition 2.2 We say that the system (2.2)-(2.4) is a negative feedback system with negative sensing (Nf-Ns system) if $\bar{\alpha}=-1$ and $\bar{\beta}=-1$.

Given our preceding definitions, the number of genes $n$, the monotonicity of each $\Phi_{j}(z)$ and the index $k$ defining $\Omega$ in $\Omega=X_{k}$ all affect the parity parameters $\bar{\alpha}$ and $\bar{\beta}$ in Definition 2.1. For instance, in the repressilator model (2.1) one can choose $\Omega \in\left\{X_{1}, X_{2}, X_{3}\right\}=\{A, B, C\}$ for the feedback to the autoinducer but only for $\Omega=A$ is the system Nf-Ns.

Ultimately, we seek to understand the dynamics of $N$ identical Nf-Ns cells. To be more explicit we let $i$ index each cell. Then, the state of each cell is given by

$$
\mathbf{x}_{i}=\left(x_{1}^{i}, X_{1}^{i}, x_{2}^{i}, X_{2}^{i}, \ldots, x_{n}^{i}, X_{n}^{i}, S_{i}\right) .
$$

Thus, $X_{j}^{i}$ is the dependent variable for the $j^{t h}$ mRNA in cell $i$. As was previously defined, $S_{i}$ is the autoinducer concentration in cell $i$. With these definitions we may now state the main result of our paper in the following Theorem.

Theorem 2.3 Let (2.2)-(2.4) be an $N f$-Ns system with $N$ identical cells. Then

1. There exists a unique equilibrium $E$ that is symmetric i.e., there exist $\bar{X}_{j}, \bar{x}_{j}$ and $\bar{S}$ such that $X_{j}^{i}=\bar{X}_{j}, x_{j}^{i}=\bar{x}_{j}, S_{i}=\bar{S}$ for all $i=1, \ldots, N$.

2. Equilibrium E can loose stability only through a Hopf bifurcation.

3. There are exactly two such Hopf bifurcations. One depends on the quorum sensing parameter $q$. The other does not.

4. A branch of synchronous periodic orbits emerge from the q-dependent Hopf bifurcation.

5. The q-independent Hopf bifurcation does not have a branch of synchronous periodic orbits.

Our results explain and significantly generalize numerical observations reported in the literature. The system (2.1) with $\Omega=A$ was studied in [16]. In their study, the quorum sensing index as defined in Definition 2.1 is $\bar{\beta}=-1$, and they found only symmetric oscillations in their numerical examinations of the Nf-Ns system.

However, when $\Omega=B$ as in $[25,26,11], C I$ promotes the autoinducer and the quorum sensing index $\bar{\beta}=1$. The resulting dynamics are significantly different. The numerical 
studies demonstrated the existence of synchronous and asynchronous oscillations as well as the coexistence of stable equilibria and periodic orbits. This seems to indicate that the systems with negative feedback $(\bar{\alpha}=-1)$ but a positive quorum sensing loop $(\bar{\beta}=1)$ are capable of bistable behavior. Later we shall demonstrate numerically for the repressilator model [16] that when $\Omega=C$, the quorum sensing loop is positive and the system can exhibit bistable behavior.

\subsection{General Framework}

In what follows, we assume cell to cell homogeneity in intracellular dynamics. Such models can be cast in a simple general framework. To do so recall the state of each cell is given by

$$
\mathbf{x}_{i}(t)=\left(x_{1}^{i}, X_{1}^{i}, x_{2}^{i}, X_{2}^{i}, \ldots, x_{n}^{i}, X_{n}^{i}, S_{i}\right) \in \mathbb{R}^{l}
$$

where $l=2 n+1$.

In the model (2.1), $l=7$, while in other repressilator model studies $l=3$ [27]. But in our general model (2.2)-(2.4) we have $l=2 n+1$ where $n$ is the number of genes. Then the state of the quorum of $N$ cells is given by

$$
\mathbf{x}=\left(\mathbf{x}_{1}, \mathbf{x}_{2}, \ldots, \mathbf{x}_{N}\right)^{T} \in \mathbb{R}^{N l}
$$

When the inter-cell coupling $q=0$, the intracellular chemical kinetics is represented by

$$
\frac{d \mathbf{x}_{i}}{d t}=f\left(\mathbf{x}_{i} ; \mu\right)
$$

for some function $f$ and parameters $\mu$. Cell to cell homogeneity is reflected in the fact $f(\mathbf{x})$ is the same for each cell $i$ in (2.5).

For quorum models where auto-inducers sense extracellular averages $(q \neq 0)$ an additional term needs to be added to (2.5):

$$
\frac{d \mathbf{x}_{i}}{d t}=f\left(\mathbf{x}_{i} ; \mu\right)+\mathcal{Q} \mathbf{x}
$$

where $\mathcal{Q}$ is a constant coupling matrix having the block structure:

$$
\mathcal{Q}=\left[\begin{array}{cccc}
C & C & \ldots & C \\
C & C & \ldots & C \\
\vdots & \vdots & \ldots & \vdots \\
C & C & \ldots & C
\end{array}\right]
$$

for some $C \in \mathbb{R}^{l \times l}$. Then the $N$-cell model is

$$
\frac{d \mathbf{x}}{d t}=F(\mathbf{x} ; \mu)=\left(\begin{array}{c}
f\left(\mathbf{x}_{1} ; \mu\right)+\mathcal{Q} \mathbf{x} \\
f\left(\mathbf{x}_{2} ; \mu\right)+\mathcal{Q} \mathbf{x} \\
\vdots \\
f\left(\mathbf{x}_{N} ; \mu\right)+\mathcal{Q} \mathbf{x}
\end{array}\right)
$$


For appropriately defined $f(\mathbf{x} ; \mu)$ and $\mathcal{Q}$, the models in $[16,25,26,11]$ and our general model (2.2)-(2.4) can all be written as (2.8). The form of (2.8) also results in linearized systems having a very specialized structure. In general the linearized system about $\overline{\mathbf{x}}(t)$ is

$$
\frac{d \mathbf{z}}{d t}=D F(\overline{\mathbf{x}}) \mathbf{z}
$$

where $D F(\overline{\mathbf{x}})$ is the Jacobian of $F$ in $(2.8)$ evaluated at $\overline{\mathbf{x}}$. For equilibria $\overline{\mathbf{x}}=\left(\overline{\mathbf{x}}_{1}, \overline{\mathbf{x}}_{2}, \ldots, \overline{\mathbf{x}}_{N}\right)^{T}$ is constant and $F(\overline{\mathbf{x}})=0$. And, for the coupling (2.7), we may represent this Jacobian in the block form

$$
\operatorname{DF}(\overline{\mathbf{x}})=\left[\begin{array}{cccc}
A_{1}+C & C & \ldots & C \\
C & A_{2}+C & \ldots & C \\
\vdots & \vdots & \ldots & \vdots \\
C & C & \ldots & A_{N}+C
\end{array}\right]
$$

where $A_{i}=D f\left(\overline{\mathbf{x}}_{i}\right)$. Further note that when the equilibrium is synchronous, all the $\overline{\mathbf{x}}_{i}$ are the same, so that $A_{i}=A$ for all $i=1,2, \ldots N$. All of these observations are true regardless of the chemical kinetics imposed via the function $f$, the number of cells $N$, the number of relevant intracellular concentrations and the number of auto-inducers which sense extracellular averages.

\section{$3 \quad$ Stability of Synchronous Equilibria}

In this section we derive linear stability criteria for synchronous equilibria $\overline{\mathbf{x}}$. The Jacobian of the linearized system (2.9) has the block structure:

$$
\operatorname{DF}(\overline{\mathbf{x}})=\left[\begin{array}{cccc}
A+C & C & \ldots & C \\
C & A+C & \ldots & C \\
\vdots & \vdots & \ldots & \vdots \\
C & C & \ldots & A+C
\end{array}\right]
$$

where $A, C \in \mathbb{R}^{l \times l}$. Its spectrum is then determined by the following theorem. Our proof is similar to the analysis in Pecora and Caroll [17].

Theorem 3.1 The characteristic equation of $M \equiv D F(\overline{\mathbf{x}})$ is

$$
P(\lambda)=Q(\lambda) R(\lambda)^{N-1}=0
$$

where $Q(\lambda)$ and $R(\lambda)$ are the characteristic polynomials of the matrices $A+N C$ and $A$, respectively. 
Proof. We first note that the block symmetric matrix $M \equiv D F(\overline{\mathbf{x}})$ can be written compactly as the matrix direct product:

$$
M=I \otimes A+\mathbf{1} \otimes C
$$

where $I \in \mathbb{R}^{N \times N}$ is the identity matrix, $\mathbf{1} \in \mathbb{R}^{N \times N}$ is the matrix of ones and $A, C \in \mathbb{R}^{l \times l}$. Since the eigenvalues of $\mathbf{1}$ are $N$ with multiplicity 1 , and 0 with multiplicity $N-1$ there exists a diagonalizing matrix $S$ such that $\mathbf{1}=S^{-1} D S$ where $D=\operatorname{diag}(N, 0,0, \ldots, 0)$. Next, we define $T=S^{-1} \otimes I_{K}$ where $I_{l} \in \mathbb{R}^{l \times l}$ is the identity matrix. Noting that $I$ is invariant with respect to similarity transformations, it is readily seen in Lemma 3.3 that

$$
M^{\prime} \equiv T^{-1} M T=I \otimes A+D \otimes C .
$$

Given $D$, the block diagonal matrix $M^{\prime}$ has one block $A+N C$ and $N-1$ blocks $A$. Therefore,

$$
\operatorname{det}(M)=\operatorname{det}(A+N C) \operatorname{det}(A)^{N-1}
$$

To find the characteristic polynomial $P(\lambda)$ of $M$ one merely replaces $A$ by $A-\lambda I$ to get

$$
\begin{aligned}
P(\lambda) & =\operatorname{det}(M-\lambda I) \\
& =\operatorname{det}(A+N C-\lambda I) \operatorname{det}(A-\lambda I)^{N-1} \\
& =Q(\lambda) R(\lambda)^{N-1}
\end{aligned}
$$

where $Q(\lambda)$ and $R(\lambda)$ are the characteristic polynomials of $A+N C$ and $A$, respectively.

Remark 3.2 Our result can be recast as an instance of a Master stability function [17] which is applicable to any type of symmetric coupling. To see this we rewrite (2.6) in the form

$$
\frac{d \mathbf{x}_{i}}{d t}=g\left(\mathbf{x}_{i} ; \mu\right)+\mathcal{R} \mathbf{x}
$$

where $\mathcal{R}$ is a constant matrix with the block structure

$$
\mathcal{R}=\left[\begin{array}{cccc}
(1-N) C & C & \ldots & C \\
C & (1-N) C & \ldots & C \\
\vdots & \vdots & \ldots & \vdots \\
C & C & \cdots & (1-N) C
\end{array}\right]
$$

and

$$
g\left(\mathbf{x}_{i} ; \mu\right)=f\left(\mathbf{x}_{i} ; \mu\right)+\mathcal{Z} \mathbf{x}
$$

with

$$
\mathcal{Z}=\left[\begin{array}{cccc}
N C & 0 & \ldots & 0 \\
0 & N C & \ldots & 0 \\
\vdots & \vdots & \ldots & \vdots \\
0 & 0 & \ldots & N C
\end{array}\right]
$$


While form (3.4) clearly shows that the diagonal $\mathbf{x}_{\mathbf{1}}=\ldots=\mathbf{x}_{\mathbf{N}}$ is invariant since $\mathcal{R} \mathbf{x}=0$ on diagonal, the form (2.6) has an advantage that it moves the quorum parameter representing cell density $q$ into the coupling term $\mathcal{Q}$. In particular, we can rewrite the equation (2.4) as

$$
\dot{S}_{i}=-k_{s 0} S_{i}+k_{s 1} \Omega_{i}-\eta(1-q) S_{i}+\frac{\eta q}{N}\left((1-N) S_{i}+\sum_{j \neq i} S_{j}\right)
$$

where the first 3 terms are parts of function $g\left(\mathbf{x}_{\mathbf{i}}\right)$ and the last term is part of the coupling matrix $\mathcal{R}$.

Lemma 3.3 Given the definition of $M, S$ and $T$ in Theorem 3.1

$$
T^{-1} M T=I \otimes A+D \otimes C .
$$

Proof. First note that the diagonalizing matrix $S$ of $\mathbf{1}$ is:

$$
S=\left[\begin{array}{rrrrr}
1 & 1 & \ldots & \ldots & 1 \\
1 & -1 & 0 & \ldots & 0 \\
1 & 0 & -1 & \ldots & 0 \\
\vdots & \vdots & \ldots & \vdots & \vdots \\
1 & 0 & \ldots & 0 & -1
\end{array}\right]
$$

We now use the distributive and mixed-product properties of the Kronecker product given $T=S^{-1} \otimes I_{K}$ :

$$
\begin{aligned}
M T & =(I \otimes A+\mathbf{1} \otimes C)\left(S^{-1} \otimes I_{K}\right) \\
& =(I \otimes A)\left(S^{-1} \otimes I_{K}\right)+(\mathbf{1} \otimes C)\left(S^{-1} \otimes I_{K}\right) \\
& =\left(I S^{-1}\right) \otimes\left(A I_{K}\right)+\left(\mathbf{1} S^{-1}\right) \otimes\left(C I_{K}\right) \\
& =S^{-1} \otimes A+\mathbf{1} S^{-1} \otimes C
\end{aligned}
$$

In a similar fashion one uses $T^{-1}=S \otimes I_{K}$ to expand

$$
T^{-1} M T=\left(S \otimes I_{K}\right) \times\left(S^{-1} \otimes A\right)+\left(S \otimes I_{K}\right)\left(\mathbf{1} S^{-1} \otimes C\right)=I \otimes A+D \otimes C
$$

Lemma 3.4 Let $\lambda_{Q}\left(\lambda_{R}\right)$ be a root of $Q(\lambda)(R(\lambda))$ and let $v_{Q}\left(v_{R}\right)$ be the corresponding eigenvector of $A+N C(A)$

$$
(A+N C) v_{Q}=\lambda_{Q} v_{Q} \quad A v_{R}=\lambda_{R} v_{R}
$$

Then 
1. the vector $\mathbf{v}_{\mathbf{Q}}:=\left(v_{Q}, v_{Q}, \ldots, v_{Q}\right)^{T}$ where $v$ repeats $N$ times is an eigenvector of (3.1) with eigenvalue $\lambda_{Q}$.

2. the vector $\mathbf{v}_{\mathbf{R}}:=\left(v_{R}, v_{R}, \ldots, v_{R}\right)^{T}$ where $v$ repeats $N$ times is NOT an eigenvector of (3.1) with eigenvalue $\lambda_{R}$.

Proof. $\quad$ Result (1) follows directly by multiplying $D F(\bar{x}) \mathbf{v}_{\mathbf{Q}}$ with $D F(\bar{x})$ defined in (3.1). To show (2) observe first that

$$
D F(\bar{x}) \mathbf{v}_{\mathbf{R}}=(v, v, \ldots, u)^{T} \quad \text { where } \quad u=\lambda_{R} v_{R}+N C v_{R} .
$$

Note that if we show that $v_{R} \notin C$, then $\mathbf{v}_{\mathbf{R}}$ is not an eigenvector of $D F(\bar{x})$, which is the statement (2).

We will now show that $v_{R} \notin C$. Since $C$ has the unique non-zero element in the lower right corner, this is equivalent to show that the last component of $v_{R}$ is non zero $\left[v_{R}\right]_{2 n+1} \neq 0$.

Assume, by contradiction, that $\left[v_{R}\right]_{2 n+1}=0$. We show that if $\left(A-\lambda_{R} I\right) v_{R}=0$ and $\left[v_{R}\right]_{2 n+1}=$ 0 , then $v_{R} \equiv 0$. Since $v_{R}$ is perpendicular to the last row of $A$, and this row has only two nonzero elements in column $2 K$ and the last column $2 n+1$, our assumption $\left[v_{R}\right]_{2 n+1}=0$ implies $\left[v_{R}\right]_{2 K}=0$. Next, consider the $2 K$-th row of $A$ which is also perpendicular to $v_{R}$ and has only two non-zero entries in columns $2 K-1$ and $2 K$. Then $\left[v_{R}\right]_{2 K}=0$ implies $\left[v_{R}\right]_{2 K-1}=0$. Realizing that each row of $A$, except the row $2 n-1$, has two entries, the same argument sequentially establishes that $\left[v_{R}\right]_{i}=0$ for all $i$.

Remark 3.5 The first result of Lemma 3.4 implies that the eigenspace at the Hopf bifurcation at $\lambda_{Q}=i \omega$ is invariant under symmetry group $S_{N}$ generated by relabelling of the oscillators. By the equivariant Hopf bifurcation theory [7, 20], for every isotropy subgroup $\Sigma \subset S_{N} \times S^{1}$ with a two dimensional fixed point space, there will be a small amplitude periodic orbit emanating from the equilibrium having $\Sigma$ as their group of symmetries. Since the two dimensional real eigenspace corresponding to $\lambda_{Q}=i \omega$ is invariant under $S_{N} \times\{1\}$, this theory guarantees existence of a branch of synchronous oscillations. Which of these branches of periodic orbits will be stable depends on delicate analysis of higher order terms of the nonlinearities, see [4].

On the other hand, the second result of Lemma 3.4 implies that the loss of stability of synchronous equilibrium through Hopf bifurcations where $\lambda_{R}=i \omega$ is a root of $R(\lambda)$ will lead to oscillations which will not be synchronous since the eigenspace at the Hopf bifurcation is not not invariant under $S_{N}$ symmetry. In this case different cells will oscillate with either different amplitudes, or different phases.

At this point we may make one very important observation. The stability of any synchronous equilibria is independent of the number of cells $N$. If the autoinducer sensing terms contain only average terms such as $\frac{\eta q}{N} \sum_{i=1}^{N} S_{i}$ then it is not hard to see, given how $C$ is defined, 
that neither $A+N C$ nor $A$ depend on $N$. Consequently, for models defined by (2.6), the stability of synchronous states and the possible emergence of oscillatory solutions from them have nothing to do with the number of cells.

\section{Nf-Ns model}

We now focus on the Nf-Ns model where $\bar{\alpha}=\bar{\beta}=-1$ in Definition 2.1. Again, the state variable for cell $i$ is

$$
\mathbf{x}_{i}=\left(x_{1}^{i}, X_{1}^{i}, x_{2}^{i}, X_{2}^{i}, \ldots, x_{n}^{i}, X_{n}^{i}, S_{i}\right)
$$

A superscript is used to index the cell while subscripts index the gene number. These conventions will be retained in subsequent calculations. Also, lowercase $x$ denote the mRNA, uppercase $X$ denote proteins and $S$ the autoinducer. Then, the quorum state $\mathbf{x}=\left(\mathbf{x}_{1}, \mathbf{x}_{2}, \ldots, \mathbf{x}_{N}\right)^{T} \in$ $\mathbb{R}^{(2 n+1) N}$, and the model (2.2)-(2.4) can be written in the compact form

$$
\frac{d \mathbf{x}}{d t}=F(\mathbf{x} ; \mu)
$$

for an appropriately defined $F$.

Again, by convention we will let overbars denote equilibria as in $F(\overline{\mathbf{x}})=0$ where $\overline{\mathbf{x}}=$ $\left(\overline{\mathbf{x}}_{1}, \overline{\mathbf{x}}_{2}, \ldots, \overline{\mathbf{x}}_{N}\right)^{T}$. Our ultimate goal is to understand how and under what circumstances synchronous oscillations might emerge in the genetic network.

In the next section, we prove the Nf-Ns model has a sole (synchronous) equilibria which can be parametrized explicitly in terms of the equilibrium value of $x_{n}$. Afterwards we prove such equilibria can only destabilize via Hopf bifurcations.

\subsection{Existence and uniqueness of synchronized equilibrium}

In this section we show the only positive equilibria of the system (2.2)-(2.4) are synchronous:

$$
\overline{\mathbf{x}}_{i}=\left(x_{1}, X_{1}, x_{2}, X_{2}, \ldots, x_{n}, X_{n}, S\right) \quad, \quad \forall i=1,2, \ldots N
$$

for some positive $x_{1}, X_{1}, x_{2}, X_{2}, \ldots, x_{n}, X_{n}$ and $S$.

Theorem 4.1 Suppose $\Phi_{j}(z)$ are monotonic for $j=1,2, \ldots n$ and that $\Gamma(z)$ is monotonically increasing. Further suppose there exists constants $a, \kappa_{1}$ and $\kappa_{2}$ such that

i) $\Phi_{j}(z) \geq 0, \Phi_{j}(0)>a>0$ and $\Phi_{j}(z)<\kappa_{1}$ for all $z \geq 0$ for all $j=1,2, \ldots n$.

ii) $\Gamma(z)>0$ for $z>0$ and $\Gamma(z)<\kappa_{2}$ for all $z \geq 0$

then (2.2)-(2.4) has a unique positive equilibria $\overline{\mathbf{x}}$ which is synchronous. 
Proof. From the form of the equations (2.2)-(2.4) it is evident that

$$
X_{j}^{i}=\frac{b_{j}}{a_{j}} x_{j}^{i}
$$

and

$$
x_{j}^{i}=\frac{1}{c_{j}} \Phi_{j}\left(X_{j-1}^{i}\right)
$$

Let

$$
\Psi_{j}(z)=\frac{b_{j}}{a_{j} c_{j}} \Phi_{j}(z)
$$

Then

$$
X_{j}^{i}=\Psi_{j}\left(X_{j-1}^{i}\right)
$$

Using this we observe that we can write for all $i$

$$
\begin{aligned}
X_{k}^{i} & =\Psi_{k} \circ \Psi_{k-1} \circ \Psi_{1}\left(\frac{b_{n}}{a_{n}} x_{n}\right)=: H\left(x_{n}\right) \\
X_{n-1}^{i} & =\Psi_{n-1} \circ \Psi_{n-2} \circ \Psi_{1}\left(\frac{b_{n}}{a_{n}} x_{n}\right)=: G\left(x_{n}\right)
\end{aligned}
$$

Next note $\dot{S}_{i}=0$ implies

$$
-\left(k_{s 0}+\eta\right) S_{i}+k_{s 1} X_{k}^{i}+\eta q S=0
$$

where the $i$-independent average ${ }^{2}$ in (4.4) is:

$$
S \equiv \frac{1}{N} \sum_{i=1}^{N} S_{i}
$$

Solving for $S_{i}$ we find

$$
S_{i}=\gamma_{1} X_{k}^{i}+\gamma_{0} S
$$

where the parameters defining $S_{i}$ are both positive and do not depend on $i$ :

$$
\gamma_{1}=\frac{k_{s 1}}{\eta+k_{s 0}} \quad, \quad \gamma_{0}=\frac{\eta q}{\eta+k_{s 0}}
$$

Collectively, using the equation

$$
0=\dot{x}_{n}^{i}=-c_{n} x_{n}^{i}+\Phi_{n}\left(X_{n-1}^{i}\right)+\Gamma\left(S_{i}\right)
$$

and plugging in expressions from (4.3) we find that all $x_{n}^{i}$ must be roots of the same function:

$$
F(x):=\Phi_{n}(G(x))+\Gamma\left(\gamma_{1} H(x)+\gamma_{0} S\right)-c_{n} x .
$$

\footnotetext{
${ }^{2}$ When the equilibria $\overline{\mathbf{x}}$ is synchronous, $S_{i}=S$ for all $i$
} 
We now show $F(x)$ has a sole root for each $S>0$ in which case all $x_{n}^{i}$ equal the same root $x_{n}$ and all equilibria of (2.1) are synchronous. First we note that because of the assumptions of nonnegativity, for all $S>0$ we have

$$
F(0)=\Phi_{n}(G(0))+\Gamma\left(\gamma_{1} H(0)+\gamma_{0} S\right) \geq \Gamma\left(\gamma_{0} S\right)>0 .
$$

Then since

$$
\lim _{x \rightarrow \infty}\left(\Phi_{n}(G(x))+\Gamma\left(\gamma_{1} H(x)+\gamma_{0} S\right)-c_{n} x\right)<\lim _{x \rightarrow \infty} \kappa_{1}+\kappa_{2}-c_{n} x
$$

it is apparent $F(x)<0$ for $x$ sufficiently large. Thus $F(x)$ has a root. This root is unique since $F$ is a monotonically decreasing function.

To see this we first observe that the sign of the derivative

$$
\operatorname{sgn} \frac{d}{d x}\left(\Phi_{n}(G(x))=\Pi_{i=1}^{n} \Phi_{i}^{\prime}=\bar{\alpha} .\right.
$$

Similarly, the sign of the derivative of the second term

$$
\operatorname{sgn} \frac{d}{d x}\left(\Gamma\left(\gamma_{1} H(x)+\gamma_{0} S\right)\right)=\operatorname{sgn} \Gamma^{\prime} \operatorname{sgn} \frac{d}{d x} H(x)=\bar{\beta}
$$

since $\operatorname{sgn} \Gamma^{\prime}=1$ by assumption. Since in Nf-Ns systems both $\bar{\alpha}=\bar{\beta}=-1, F(x)$ is decreasing and has a sole positive root for each $S>0$.

\subsection{Equilibria destabilization for a general case}

Given Theorem 3.1 the stability of $\overline{\mathbf{x}}$ will depend only on the spectra of $A$ and $A+N C$, which differ only in the lower right single element. We define

$$
A_{\zeta}=\left[\begin{array}{rrrrrrrc}
-c_{1} & 0 & 0 & 0 & 0 & \ldots & \Phi_{1}^{\prime}\left(x_{n}\right) & 0 \\
b_{1} & -a_{1} & 0 & 0 & 0 & \ldots & 0 & 0 \\
0 & \Phi_{2}^{\prime}\left(x_{1}\right) & -c_{2} & 0 & 0 & \ldots & 0 & 0 \\
0 & 0 & b_{2} & -a_{2} & 0 & \ldots & 0 & 0 \\
0 & 0 & 0 & \Phi_{3}^{\prime}\left(x_{2}\right) & -c_{3} & \ldots & 0 & 0 \\
\vdots & & \vdots & & \vdots & & \vdots & \\
0 & 0 & 0 & \Phi_{n}^{\prime}\left(x_{n-1}\right) & -c_{n} & \ldots & 0 & \Gamma^{\prime}(S) \\
0 & 0 & 0 & 0 & 0 & \ldots & -a_{n} & 0 \\
0 & k_{s} & 0 & 0 & 0 & \ldots & 0 & -\zeta
\end{array}\right]
$$

whose characteristic polynomial is

$$
P_{\zeta}(\lambda) \equiv \operatorname{det}\left(A_{\zeta}-\lambda I\right) \quad, \quad \zeta>0
$$


In matrix $A_{\zeta}$ we placed $k_{s 1}$ in the second column - this correspond to $k=1$ where variable $X_{1}$ is involved in the quorum sensing feedback loop. For general $k$ the number $k_{s 1}$ will be in the column $2 k$.

Then if we define

$$
\zeta_{1}=k_{s 0}+(1-q) \eta \quad, \quad \zeta_{2}=k_{s 0}+\eta
$$

the respective characteristic polynomials of $A+N C$ and $A$ in Theorem 3.1 are:

$$
\begin{aligned}
& Q(\lambda)=P_{\zeta_{1}}(\lambda) \\
& R(\lambda)=P_{\zeta_{2}}(\lambda)
\end{aligned}
$$

where $\zeta_{1}<\zeta_{2}$ and only $Q(\lambda)$ depends on the quorum sensing parameter $q$. It therefore suffices to examine roots of the polynomial $P_{\zeta}(\lambda)$.

The sparsity of $A_{\zeta}$ helps in the computation of $P_{\zeta}(\lambda)$. We let $M_{i, j}$ be the sub-matrix obtained by deleting the $i^{t h}$ row and $j^{\text {th }}$ column of $A_{\lambda} \equiv A_{\zeta}-\lambda I$. Then the Laplace expansion of $A_{\lambda}$ about the last row yields:

$$
P_{\zeta}=-(\lambda+\zeta)\left|M_{2 l+1,2 l+1}\right|-k_{s 1}\left|M_{2 l+1,2 k}\right|
$$

where $k$ is the index of the variable $X_{k}$ which is involved in quorum sensing loop.

We first indicate computation of $\left|M_{2 l+1,2 l+1}\right|$. This minor has elements only on the two diagonals of the matrix: the main diagonal, and the lower diagonal, into which we include the element $\Phi_{1}^{\prime}\left(x_{n}\right)$ in the upper right corner of $M_{2 l+1,2 l+1}$. This structure implies that there are exactly two nonzero products in the determinant calculation that multiply elements along these two diagonals. Therefore

$$
\begin{aligned}
\left|M_{2 l+1,2 l+1}\right| & =\Pi_{i=1}^{n}\left(-c_{i}-\lambda\right)\left(-a_{i}-\lambda\right)-\Pi_{i=1}^{n} b_{i} \Pi_{i=1}^{n} \Phi_{i}^{\prime} \\
& =\Pi_{i=1}^{n}\left(c_{i}+\lambda\right)\left(a_{i}+\lambda\right)-\bar{\alpha} \Pi_{i=1}^{n} b_{i}\left|\Pi_{i=1}^{n} \Phi_{i}^{\prime}\right|
\end{aligned}
$$

where in the last equality we used definition of $\bar{\alpha}$.

The calculation of the term $\left|M_{2 l+1,2 k}\right|$ is more involved since we will need to discuss its dependence on $k$. We perform the calculation on the matrix (4.6) where $k=1$; we then make few remark on the general case. Note that there is unique nonzero element in the second row of $M_{2 l+1,2}$; so every nonzero term in the determinant must contain the entry $b_{1}$. Expanding around pivot $b_{1}$ in turn forces selection of $\Phi_{1}^{\prime}\left(x_{n}\right)$ as the next pivot in the first row.

For general $k$, an analogous process forces selection of (in order)

$$
b_{k}, \Phi_{k-1}^{\prime}, b_{k-1}, \Phi_{k-1}^{\prime}, \ldots, b_{1}, \Phi_{1}^{\prime} .
$$

After this backward sweep, we go back to the example (4.6) and expand the matrix forward along the diagonal from column 2 . In the row 3 we need to expand around $\left(-c_{2}-\lambda\right)$, which 
then forces $\left(-a_{2}-\lambda\right)$. It follows that in order to get a nonzero product we need to expand along the diagonal elements

$$
\left(-c_{2}-\lambda\right),\left(-a_{2}-\lambda\right), \ldots\left(-c_{n-1}-\lambda\right),\left(-a_{-1}-\lambda\right) .
$$

However, the only nonzero element in the last column of $M_{2 l+1,2}$ is $\Gamma^{\prime}(S)$. Therefore, in order to ensure nonzero product in the determinant we need to pivot on $\Gamma^{\prime}(S)$ and, finally, include $b_{n}$.

Therefore, for general $k$ we have

$$
\begin{aligned}
\left|M_{2 l+1,2 k}\right| & =\Gamma^{\prime}(S) b_{n}\left(\Pi_{i=k}^{n-1}\left(-c_{i}-\lambda\right)\left(-a_{i}-\lambda\right)\right) \Pi_{i=1}^{k} b_{i} \Pi_{i=1}^{k-1} \Phi_{i}^{\prime} \\
& =\Gamma^{\prime}(S) b_{n}\left(\Pi_{i=k}^{n-1}\left(c_{i}+\lambda\right)\left(a_{i}+\lambda\right)\right) \Pi_{i=1}^{k} b_{i} \Pi_{i=1}^{k-1} \Phi_{i}^{\prime}
\end{aligned}
$$

Note that since

$$
\bar{\alpha}=\operatorname{sgn} \Pi_{i=1}^{n} \Phi_{i}^{\prime}=\operatorname{sgn}\left(\Pi_{i=1}^{k-1} \Phi_{i}^{\prime} \Pi_{i=k}^{n} \Phi_{i}^{\prime}\right)=\operatorname{sgn}\left(\Pi_{i=1}^{k-1} \Phi_{i}^{\prime}\right) \bar{\beta}
$$

and $\bar{\alpha}=\bar{\beta}=-1$ in the Nf-Ns case, we have

$$
\Pi_{i=1}^{k-1} \Phi_{i}^{\prime}>1
$$

Using these results we compute $\bar{P}(\lambda)=-P_{\zeta}(\lambda)$

$$
\bar{P}(\lambda)=(\lambda+\zeta) \Pi_{i=1}^{n}\left(c_{i}+\lambda\right)\left(a_{i}+\lambda\right)+\rho_{1}(\lambda+\zeta)+\rho_{2}\left(\Pi_{i=k}^{n-1}\left(c_{i}+\lambda\right)\left(a_{i}+\lambda\right)\right)
$$

where

$$
\begin{aligned}
& \rho_{1}=-\bar{\alpha} \Pi_{i=1}^{n} b_{i}\left|\Pi_{i=1}^{n} \Phi_{i}^{\prime}\right|>0 \\
& \rho_{2}=\Phi_{i}^{\prime} k_{s 1} \Gamma^{\prime}(S) b_{n} \Pi_{i=1}^{k} b_{i} \Pi_{i=1}^{k-1} \Phi_{i}^{\prime} k_{s 1} \Gamma^{\prime}(S)>0
\end{aligned}
$$

Note we negated $P_{\zeta}$ to remove several minus signs. Again, all parameters are positive. Importantly, this implies

$$
\bar{P}(\lambda)>0 \quad, \quad \forall \lambda>0
$$

for all positive model parameters $\mu$. This means that regardless of parameter values $\mu$, the Jacobian $\operatorname{DF}(\overline{\mathbf{x}})$ can not have positive real eigenvalues . Consequently, destabilization of equilibria can occur only as a result of a Hopf bifurcation. In so much as we are interested in the emergence of oscillations we next seek to locate such Hopf points.

We rewrite (4.9) in the following way

$$
\bar{P}(\lambda)=(\lambda+\zeta)\left[p^{n}(\lambda)+\rho_{1}\right]+\rho_{2} p^{k}(\lambda)
$$

where

$$
p^{s}(\lambda):=\Pi_{i=1}^{s}\left(c_{i}+\lambda\right)\left(a_{i}+\lambda\right) .
$$


To find Hopf point loci, we exploit the fact that $\bar{P}(\lambda)$ defined in (4.10) depends linearly on $\rho_{1}$ and $\rho_{2}$. At Hopf points $\lambda=i y, y \in \mathbb{R}$ so the two equations

$$
\begin{aligned}
& \mathbb{R} e(\bar{P}(i y))=\mathcal{P}_{1}\left(y, \rho_{1}, \rho_{2}\right)=0 \\
& \mathbb{I} m(\bar{P}(i y))=\mathcal{P}_{2}\left(y, \rho_{1}, \rho_{2}\right)=0
\end{aligned}
$$

represent a solvable linear system whose solution $\left(\rho_{1}, \rho_{2}\right)$ is parametrized by the local frequency $y$.

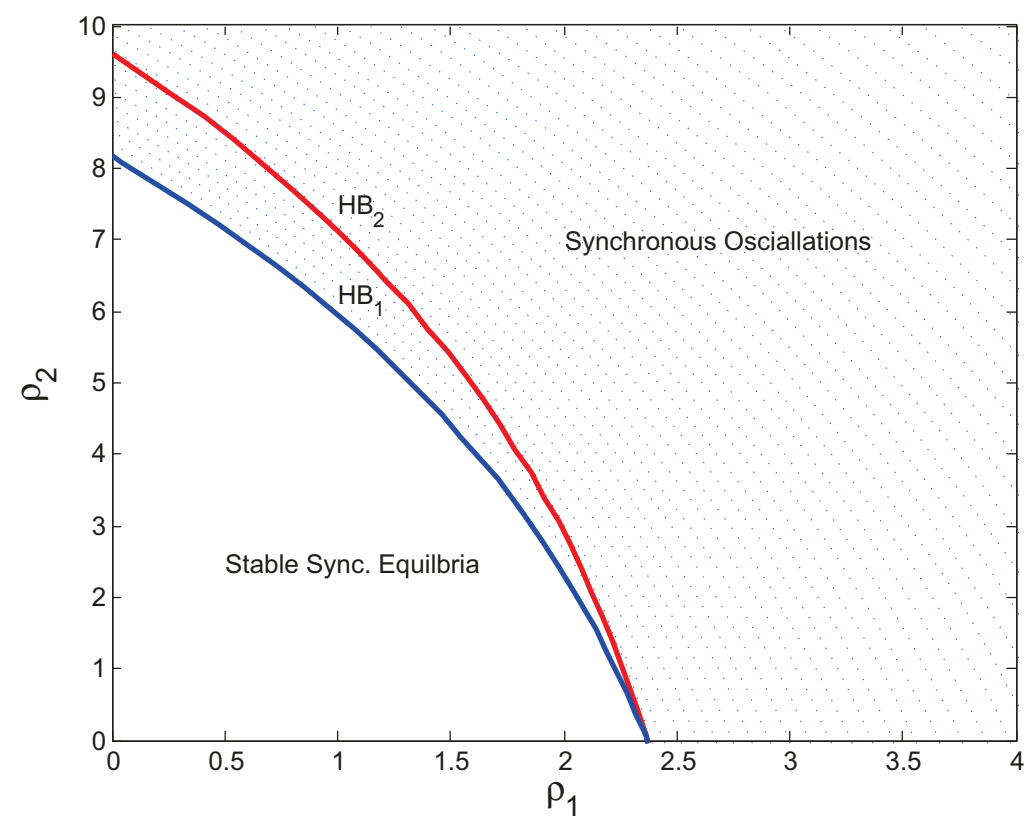

Figure 4: Shows the loci of Hopf curves $H B_{i}$ of the repressilator model [16] derived by solving the system (4.11)-(4.12) for $\zeta=\zeta_{i}, i=1,2$. The blue $H B_{1}$ curve is the locus of Hopf points that depend on the quorum sensing parameter $q$. In contrast, the red $H B_{2}$ curve is the locus of Hopf points that do not depend on $q$. Above $H B_{1}$ the sole (synchronous) equilibria is unstable (shaded region). For $\left(\rho_{1}, \rho_{2}\right)$ below this curve, the sole equilibria is stable. Parameter values needed to compute Hopf curves are $\left(\beta, k_{s 0}, k_{s 1}, \eta, q\right)=(1,1,0.01,2,0.2)$.

We demonstrate this procedure for finding Hopf loci on the Garcia-Ojalvo et al. represillator model [16] which belongs to the Nf-Ns class of models. The results of these calculations are used in Figure 4 to plot the two Hopf Loci curves defined by $\zeta=\zeta_{i}, i=1,2$. In addition to these two curves (4.11)-(4.12) was solved using $\lambda=x+i y$ for various positive $x$ values. Such curves correspond to locations within the $\left(\rho_{1}, \rho_{2}\right)$-plane where the sole equilibria are unstable (shaded region). For the parameters used in Figure 4 we observe:

- Only the Hopf locus $H B_{1}$ separates regions of equilibria stability. Moreover, only this curve depends explicitly on $q$ through $\zeta_{1}=k_{s 0}+(1-q) \eta$. The destabilization of the 
equilibria at $H B_{1}$ occurs at smaller $\rho_{k}$ values than those on $H B_{2}$ as $\rho_{k}$ is increased $(k=1,2)$. Thus, in this sense and this case, the quorum sensing mechanism causes an early onset of system oscillations $\left(H B_{1}\right.$ and $H B_{2}$ coincide when $\left.q=0\right)$.

- The Hopf locus $H B_{1}$ corresponds to roots of polynomial $Q(\lambda)$ which by Remark 3.5 corresponds to birth of synchronous oscillations i.e oscillations that are identical in each cell.

We conclude this section by noting the emergence of Hopf points in other model parameters can also be found but must be located numerically. One such result is shown in Figure 5. Two analogous curves for $H B_{1}$ and $H B_{2}$ are shown in the $(\alpha, \beta)$-plane. In the inlay, a bifurcation diagram of the $A_{1}$ component versus $\alpha$ for $\beta=1$ and $N=2$ cells is shown. The emergence of periodic orbits (and their stability) is clearly evident. Lastly, note that for each $\alpha$ the model has at most one (synchronous) attractor. As a reminder it is for this reason we refer to this class of models as monostable.

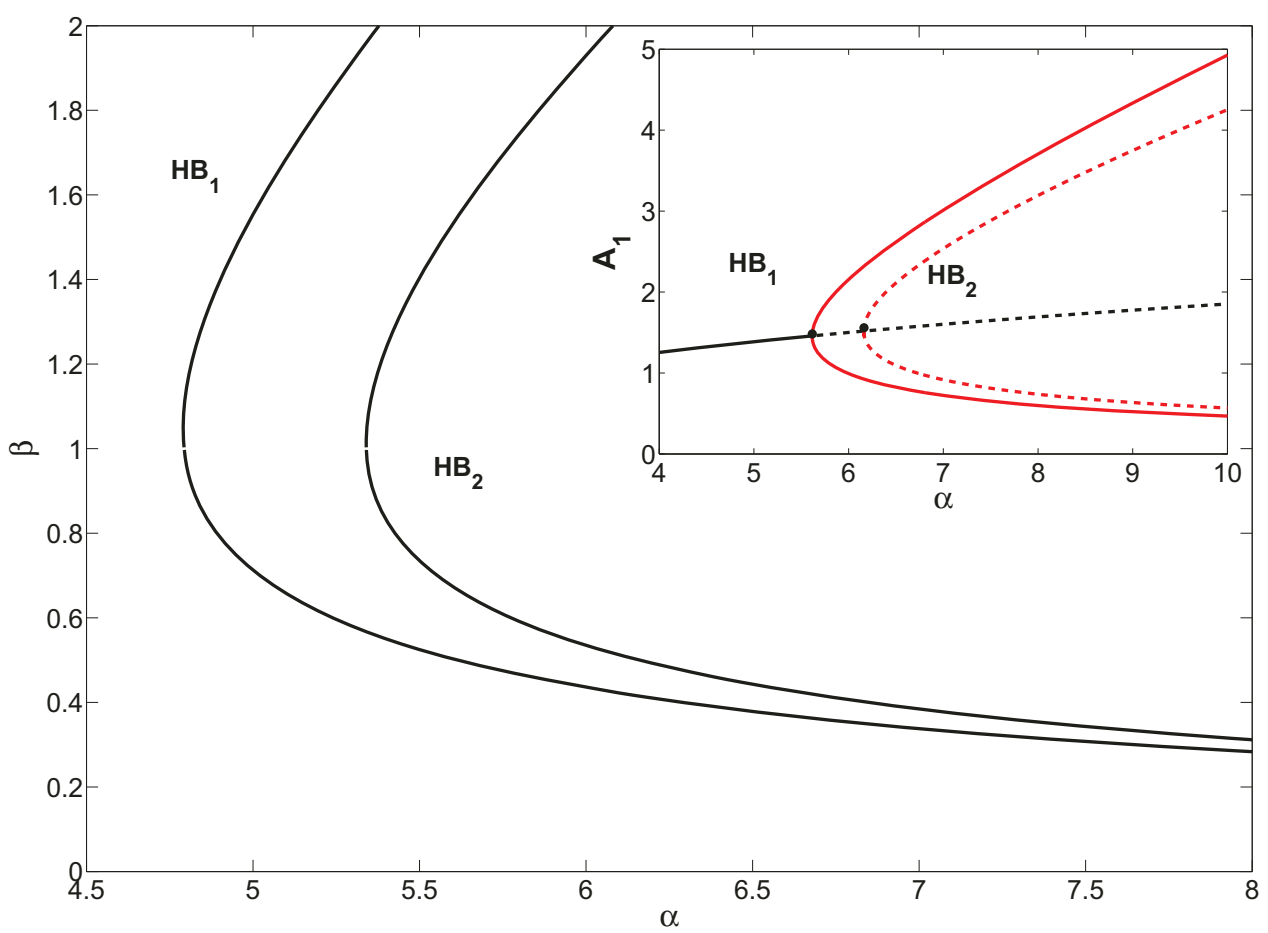

Figure 5: Shows Hopf Bifurcation loci in an $N=2$ cell model. The criticality of the Hopf bifurcations is indicated in the inlay for $\beta=1$. For all $\alpha$ shown the model is monostable. Parameter values used were $\left(n, m, \kappa, k_{s 0}, \eta, k_{s 1}, q\right)=(2,1,25,1,2,0.01,1)$ 


\section{$5 \quad$ Bistable Models}

In this section we analyze the effects of different positive feedback mechanisms for the quorum sensing. When the system is not Nf-Ns, multiple equilibria are possible and the dynamics can be much more complicated. We illustrate such possibilities on two simple variants (see Figure 1) of the repressilator model (2.1) of Ojalvo [16]. In the original model [16], tetR $(\Omega=A)$ provided positive feedback. Alternately, in $[25,26,11]$ it was assumed cI provided the feedback $(\Omega=B)$. In this case, the sole change to the model was the second term of the auto-inducer equation. For clarity, these two models are defined by

$$
\begin{aligned}
\dot{a}_{i} & =-a_{i}+\Phi\left(C_{i}\right) & & (\text { tet } R) \\
\dot{A}_{i} & =-\beta A_{i}+\beta a_{i} & & (\text { TetR }) \\
\dot{b}_{i} & =-b_{i}+\Phi\left(A_{i}\right) & & (c I) \\
\dot{B}_{i} & =-\beta B_{i}+\beta b_{i} & & (\text { CI }) \\
\dot{c}_{i} & =-c_{i}+\Phi\left(B_{i}\right)+\Gamma\left(S_{i}\right) & & (\text { lacI }) \\
\dot{C}_{i} & =-\beta C_{i}+\beta c_{i} & & (\text { LacI })
\end{aligned}
$$

where, when $\Omega=B$, the autoinducer equation is

$$
\dot{S}_{i}=-\left(k_{s 0}+\eta\right) S_{i}+k_{s 1} B_{i}+\frac{\eta q}{N} \sum_{i=1}^{N} S_{i}
$$

When $\Omega=C$, the autoinducer equation is:

$$
\dot{S}_{i}=-\left(k_{s 0}+\eta\right) S_{i}+k_{s 1} C_{i}+\frac{\eta q}{N} \sum_{i=1}^{N} S_{i}
$$

Note that we use the lower case variable names $a_{i}, b_{i}, c_{i}$ in (5.1) for time dependent protein concentrations. This should not be confused with the use of the same letters as parameter names in the generalized Nf-Ns models.

At least for (5.2), such minor differences were shown to profoundly change the behavior of the network. Here we seek a unified theory to provide a partial explanation of such differences. Firstly we use the same procedure outlined in Section 4 to determine equilibria values. Regardless of whether $A, B$ or $C$ provides positive feedback

$$
a_{i}=\Phi\left(c_{i}\right) \quad b_{i}=\Phi^{2}\left(c_{i}\right)
$$

Then, at equilibrium,

$$
\begin{aligned}
S_{i} & =\gamma_{1} \Phi\left(c_{i}\right)+\gamma_{0} S \\
S_{i} & =\gamma_{1} \Phi^{2}\left(c_{i}\right)+\gamma_{0} S \\
S_{i} & =\gamma_{1} c_{i}+\gamma_{0} S
\end{aligned}
$$


in which case $c_{i}$ must be the a root of (respectively)

$$
\begin{aligned}
& F_{a}(c) \equiv \Phi^{3}(c)+\Gamma\left(\gamma_{1} \Phi(c)+\gamma_{0} S\right)-c \\
& F_{b}(c) \equiv \Phi^{3}(c)+\Gamma\left(\gamma_{1} \Phi^{2}(c)+\gamma_{0} S\right)-c \\
& F_{c}(c) \equiv \Phi^{3}(c)+\Gamma\left(\gamma_{1} c+\gamma_{0} S\right)-c
\end{aligned}
$$

In all three cases $\Phi^{3}(c)-c$ is an initially (strictly) positive decreasing function which is negative for all $c$ sufficiently large. Thus the monotonicity properties of $F_{x}$ are relegated to those of the second (middle) term of $F_{x}$. In Theorem 4.1 we proved $F_{a}^{\prime}(c)<0$ for all $c$, a fact which depended on the argument of $\Gamma$ being a decreasing function of $c$. As a consequence, $F_{a}$ had a sole root and only synchronous equilibria were possible. Synchronous equilibria are also possible in the other cases. Such equilibria have $S_{i}=S$ for all $i$ where from (5.4)-(5.9) we deduce

$$
\begin{aligned}
S & =\gamma \Phi(c) \\
S & =\gamma \Phi^{2}(c) \\
S & =\gamma c
\end{aligned}
$$

\section{where}

$$
\gamma=\frac{\gamma_{1}}{1-\gamma_{0}}
$$

and $c$ is any root of

$$
\begin{aligned}
& F_{a}(c)=\Phi^{3}(c)+\Gamma(\gamma \Phi(c))-c \\
& F_{b}(c)=\Phi^{3}(c)+\Gamma\left(\gamma \Phi^{2}(c)\right)-c \\
& F_{c}(c)=\Phi^{3}(c)+\Gamma(\gamma c)-c
\end{aligned}
$$

respectively. In Figure 6 we show graphs of $F_{b}(c)$ and $F_{c}(c)$ for various $S$ values demonstrating bistability is possible for both mechanisms. In both cases, $F_{x}(c ; S)=0$ for three distinct roots

$$
c=\bar{c}_{1}(S), \bar{c}_{2}(S), \bar{c}_{3}(S) \quad, \quad S \in\left(S_{-}, S_{+}\right)
$$

The red curves in Figure 6 show the saddle node bifurcations of $F_{x}(c ; S)=0$, namely $S=S_{-}$ and $S=S_{+}$. Only for $S \in\left(S_{-}, S_{+}\right)$can $F_{x}(c ; S)=0$ have three roots. Consequently, the associated systems have three synchronous equilibria $c_{i}=\bar{c}_{k}(S), k=1,2,3$.

Additional asynchronous equilibria may exist but they must satisfy a consistency condition. To define this condition we note system equilibrium are uniquely determined by:

$$
\mathbf{c} \equiv\left(\bar{c}_{m_{1}}(S), \bar{c}_{m_{2}}(S), \ldots \bar{c}_{m_{N}}(S)\right) \quad, \quad m_{k} \in\{1,2,3\}
$$


a)

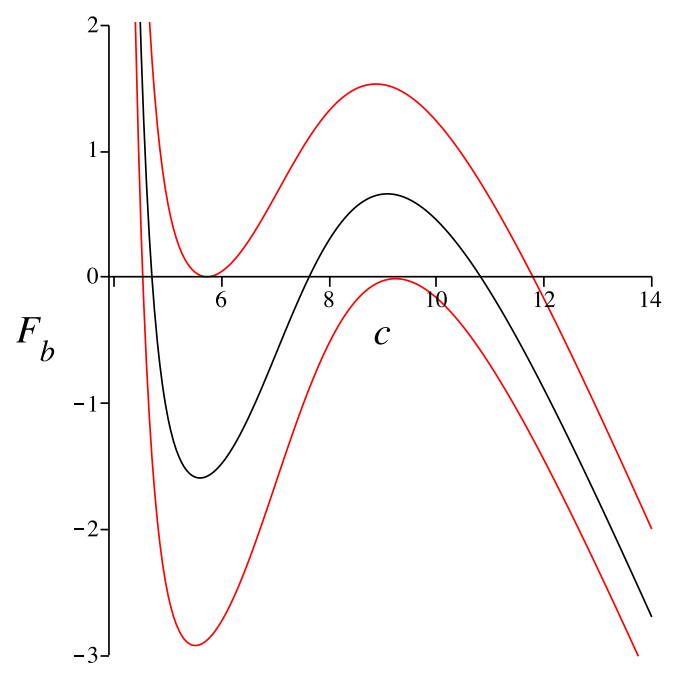

b)

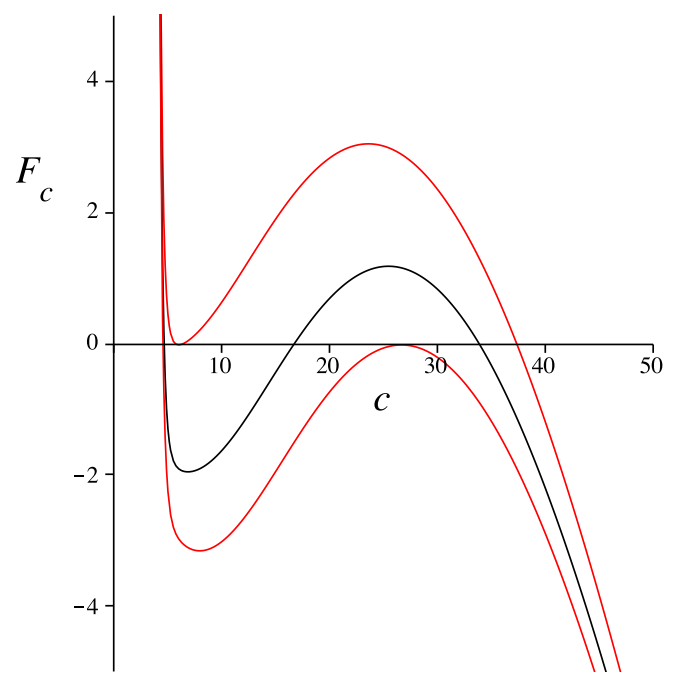

Figure 6: Shows plots of $F_{b}(c ; S)$ (left) and $F_{c}(c ; S)$ (right) for various values of autoinducer concentration $S$. In both cases, saddle-node bifurcations are evident (red). For $S \in\left(S_{-}, S_{+}\right)$, both functions have three roots. Otherwise, $F_{b}$ and $F_{c}$ have single roots. Parameter values the $B$-feedback in a) were $\mu=\left(\alpha, \beta, \kappa, n, m, k_{s 0}, k_{s 1}, \eta, q\right)=$ $(216,0.1,25,2.6,1,1,0.01,2,0.3)$. For $C$-feedback the parameter values were $\mu=$ $\left(\alpha, \beta, \kappa, n, m, k_{s 0}, k_{s 1}, \eta, q\right)=(216,1.0,55,2.6,2,15,0.6,2,0.4)$

For this illustration we consider the case where $C$ provides the positive feedback to the autoinducer so that $S_{i}$ satisfy (5.3). Then, adding (5.6) in $i=1,2, \cdots N$ and dividing by the number of cells $N$ we find $S$ must be equal to the average

$$
S=\gamma\left(\frac{N_{1}}{N} \bar{c}_{1}(S)+\frac{N_{2}}{N} \bar{c}_{2}(S)+\frac{N_{3}}{N} \bar{c}_{3}(S)\right) \equiv \Lambda_{\left(N_{1}, N_{2}, N_{3}\right)}(S)
$$

where $N_{k}$ is the number of components with $\bar{c}_{k}(S)$. Alternately, if $B$ provides the autoinducer feedback, averaging (5.5) yields the consistency condition

$$
S=\gamma\left(\frac{N_{1}}{N} \Phi^{2}\left(\bar{c}_{1}(S)\right)+\frac{N_{2}}{N} \Phi^{2}\left(\bar{c}_{2}(S)\right)+\frac{N_{3}}{N} \Phi^{2}\left(\bar{c}_{3}(S)\right)\right) \equiv \Lambda_{\left(N_{1}, N_{2}, N_{3}\right)}(S)
$$

In Figure 7 we show a plot of $\Lambda(S)$ versus $S$ for $N=3$ cells and all permutations of $\left(N_{1}, N_{2}, N_{3}\right)$. Since $B$ provides the autoinducer feedback, the consistency condition (5.11) defines $\Lambda(S)$. Then, each intersection point in Figure 6 corresponds to a value of $S$ at which 


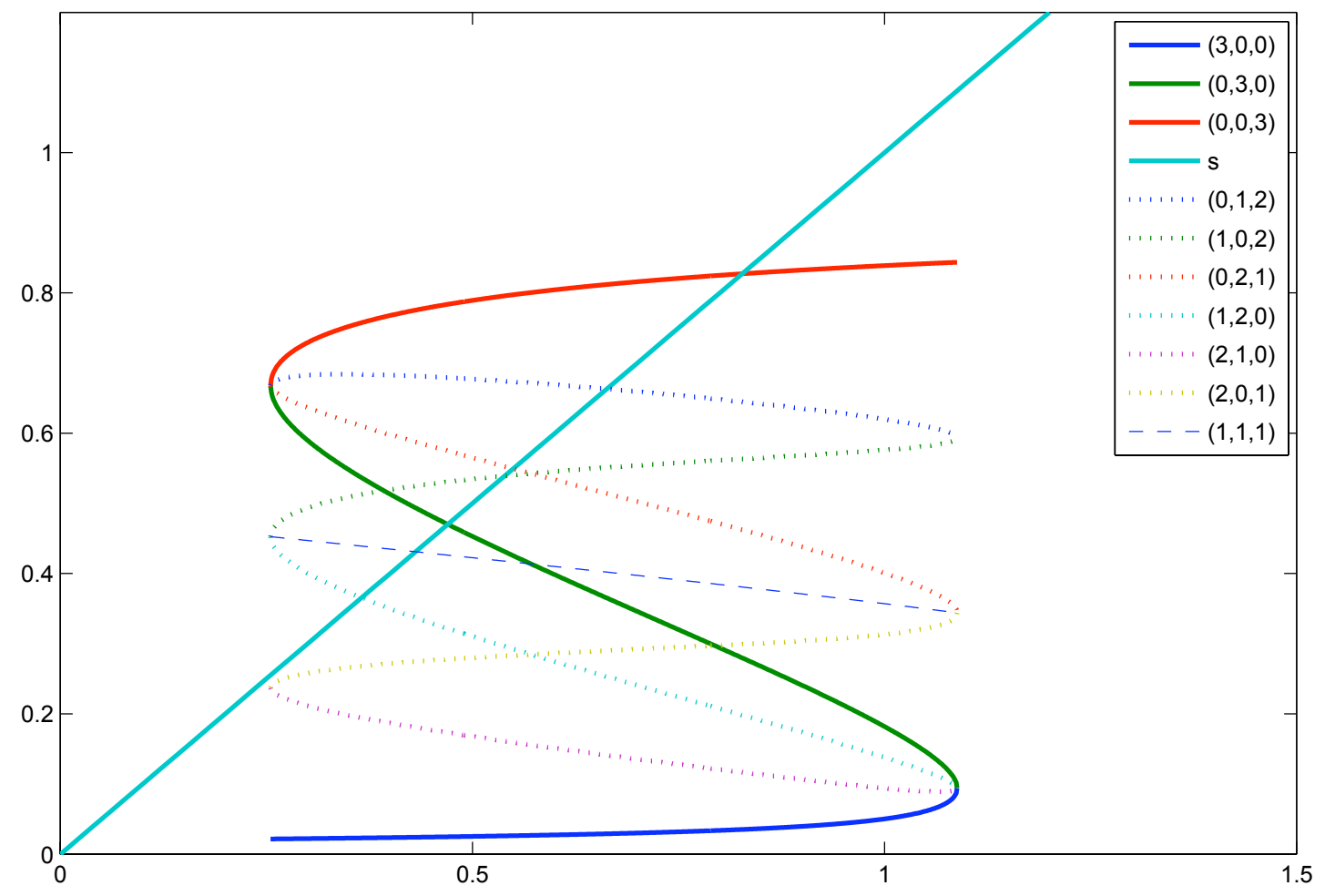

Figure 7: Asynchronous equlibria with $B$ feedback. Shows a plot of $\Lambda(S)$ for all different $\left(N_{1}, N_{2}, N_{3}\right)$ values versus $S$ (straight line). The $S$ values at intersection points are the only $S$ values possible for (all) equilibria.

the system has an equilibria. Recalling $S$ is the average autoinducer concentration at equilibria, the individual cell equilibria values $S_{i}$ are most generally not the same. Intersections above with the bold lines indicate the three possible synchronous equilibria. All others are asynchronous. We see in Figure 7 that many of these (dashed lines) also undergo saddle node bifurcations. Regardless, given (5.11), the $S$ values of all intersection points lie within $\left(S_{-}, S_{+}\right)$. Additional Hopf bifurcations which emerge from these new equilibria must also occur for $S$ within this same interval. It is therefore of great interest to understand when such intervals exist and how broad they are. 


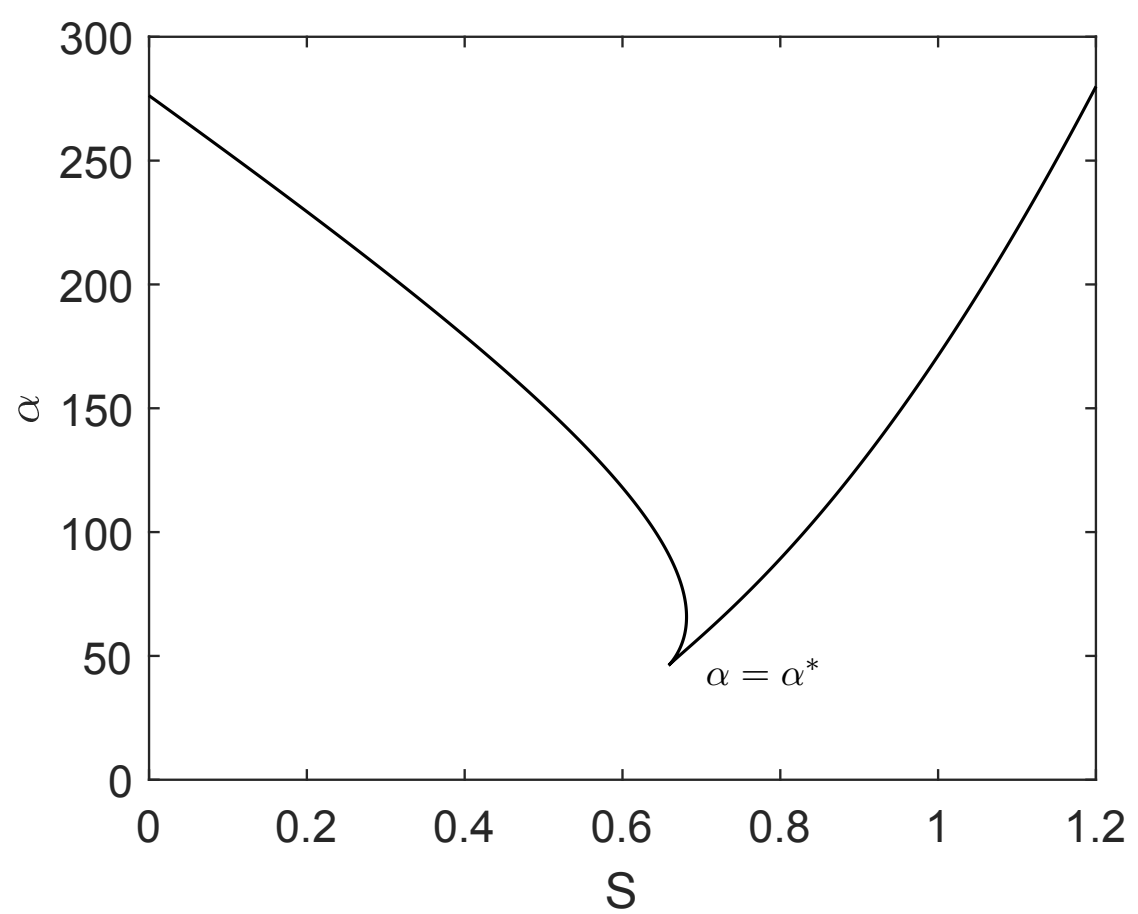

Figure 8: Numerical continuation of the interval $\left(S_{-}, S_{+}\right)$illustrated in Figure 5 . As the parameter $\alpha$ is decreased the interval disappears in a cusp bifurcation when $S_{-}=S_{+}$. At low $\alpha$ the system is monostable. Parameter values for the computations were $\mu=$ $\left(\alpha, \beta, \kappa, n, m, k_{s 0}, k_{s 1}, \eta, q\right)=(*, 0.1,25,2.6,1,1,0.01,2,0.3)$.

In Figure 8 we show the numerical continuation of the interval $I=\left(S_{-}, S_{+}\right)$as a function of the activation parameter $\alpha$. The interval width decreases with $\alpha$ and ultimately disappears in a cusp bifurcation at $\alpha=\alpha^{*}$. Consequently, the system becomes monostable for $\alpha<\alpha^{*}$. For $\alpha>\alpha^{*}$ there are a multitude of asynchronous equilibria as predicted in Figure 7 . One example showing this is presented in Figure 9 where model parameter values are set to those in Figure 7 while varying the quorum sensing parameter $q$. Even though the number of cells $N=3$ is small there are numerous stable and unstable branches of equilibria, as well as saddle node and Hopf bifurcations. Numerical studies show such bifurcation diagrams to be typical of the bistable $B$ feedback variant (Koseska et. al. [11]). 


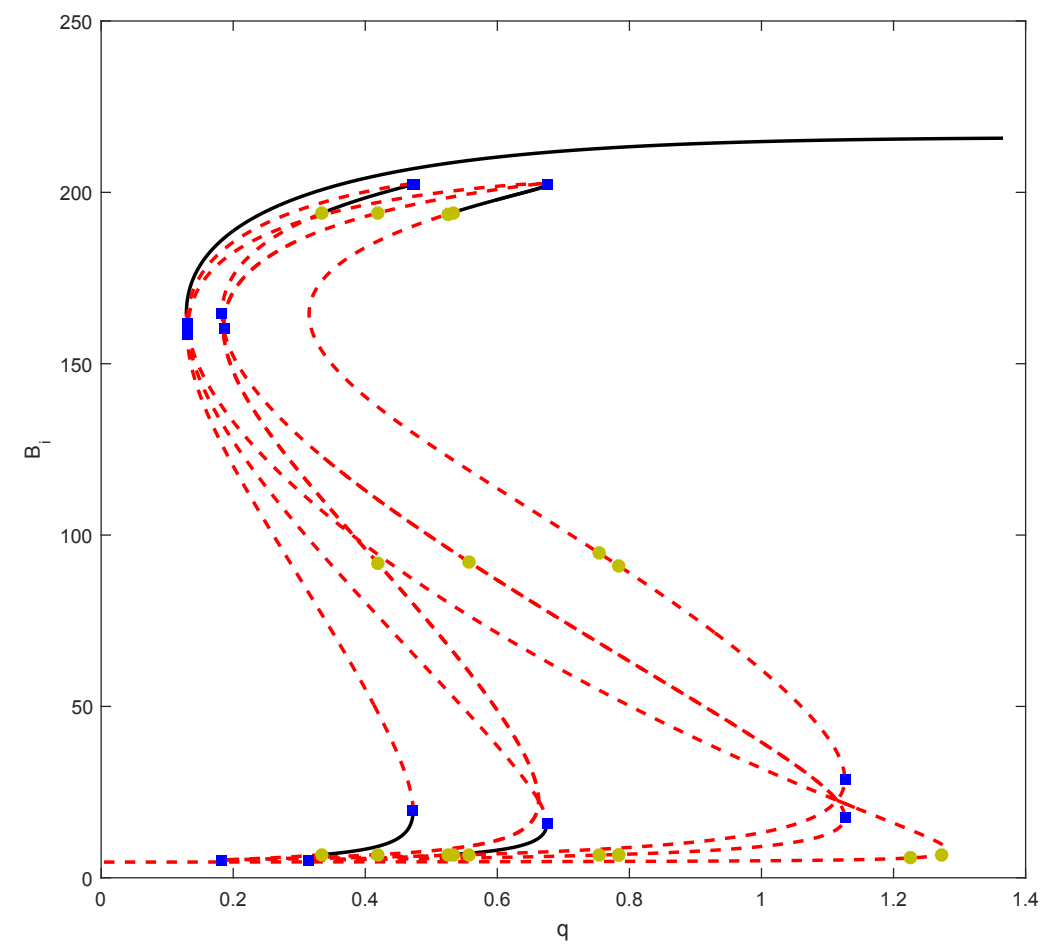

Figure 9: $B_{i}$ versus quorum sensing parameter $q$ for $\alpha>\alpha^{*}$ value where multi-stability is possible. Multiple stable (solid) and unstable (dashed) equilibria branches are shown. Saddle node (squares) and Hopf bifurcation (circles) points are numerous even in this $N=3$ cell computation.

\section{Conclusion}

The major contribution of this paper is development of mathematical theory that starts to explain why there are substantial differences in global behavior of diffusively coupled cyclic feedback systems. We define Nf-Ns (negative feedback, negative sensing) systems where the global behavior is characterized by a unique (synchronous) equilibrium which can destabilize only via a Hopf Bifurcation. These results apply to models with $n$ genes and do not depend on the number of cells $N$ in the quorum. Moreover, since the Garcia-Ojalvo et. al. [16] repressilator model with quorum sensing is an Nf-Ns system, the same results hold true for it.

The proof of the stability of equilibria in such systems relied heavily on Theorem 3.1 which effectively block diagonalizes the Jacobian. In Theorem 3.1 the associated characteristic polynomial $P(\lambda)=Q(\lambda) R(\lambda)^{N-1}>0$ hence the (unique) synchronous equilibria can destabilize only via a Hopf bifurcation. The polynomial $Q(\lambda)$ depends on the quorum sensing parameter $q$ whereas $R(\lambda)$ does not. The (sole) imaginary roots of these polynomials characterize two fundamentally different types of Hopf bifurcations. Using symmetry arguments we show that only from one of these, called $H B_{1}$, can a branch of synchronous oscillations emerge.

Our analysis also leads to a new method of numerically computing Hopf bifurcation branches in such models. We applied this method to locate these branches for a coupled repressilator model [16], which is one particular example an Nf-Ns system. 
In Figure 4, the loci for the Hopf bifurcations in the $\left(\rho_{1}, \rho_{2}\right)$-parameter space was found for the Garcia-Ojalvo et. al. [16] model. There the Hopf branch $H B_{1}$ divides the space into two regions, one where only the synchronous equilibria is stable and one where only the emergent oscillations are stable. Thus, the system is only monostable. We also note that the Hopf locus $H B_{1}$ separating different regions of stability ${ }^{3}$ was the only branch whose position in the parameter space depends on the quorum sensing parameter $q$ and the emergent periodic orbits represent synchronous oscillations.

In Section 5 we examined $K=3$ variants of [16] with $\Omega=B$ and $\Omega=C$ providing feedback to the autoinducer. As these variants were not Nf-Ns systems, multiple equilibria are possible. For both variants, the existence of system equilibria reduced to finding roots of a scalar function $F_{x}$. For some parameter values $F_{x}(S)$ had a cubic shape allowing for multiple equilibria. This is clearly evident in Figure 6a)-b) where multiple roots exist only for $S \in\left(S_{-}, S_{+}\right)$where $S$ is the average autoinducer concentration $S$. Individual system equilibria are neatly portrayed in Figure 7 as intersection points. Such points yield the $S$ values where equilibria must satisfy respective consistency conditions.

Though no analytical results for equilibria stability were presented, subsequent numerical results in Figure 8 indicate that two or more such equilibria can be stable and coexist. It is for this reason such systems are referred to as bistable. In reality such systems are multi-stable and can have a plethora of Hopf and saddle node bifurcations as well as synchronous and asynchronous equilibria. In these cases the global dynamics are extremely complicated and have numerous different (asymmetric) stable solutions. These are hard to categorize since their basins of attraction are not known apriori (see Ulner et. al. [26] and Koseska et. al. [11] for a couple of examples of studies in this regard). We should stress that such systems are also subject to threshold behavior, as with other bistable systems. For instance, in Figure 8 we demonstrate that interval $\left(S_{-}, S_{+}\right)$of $S$ for which the system has multiple equilibria disappears in a cusp bifurcation at $\alpha=\alpha^{*}$. For $\alpha<\alpha^{*}$ the system is monostable and complex system dynamics can therefore exist only if $\alpha>\alpha^{*}$.

It remains an open question as to why there is such a fundamental difference in the behavior of Nf-Ns and Nf-Ps systems, i.e., those where the diffusive coupling is mediated through a positive loop. This paper presents an extension of work done more than two decades ago that characterized global dynamics of both positive and negative feedback systems $[13,8,9]$. While the numerical results suggest that the complete characterization of the global dynamics of Nf-Ps systems may not be possible, our results on monostability of Nf-Ns systems represents a first step in characterization of global dynamics of these systems.

\footnotetext{
${ }^{3}$ of equilibria and periodic orbits
} 


\section{Acknowledgment:}

We would like to thank anonymous referees for thoughtful suggestions that substantially improved the paper.

\section{References}

[1] Buse, O., Kuznetsov,A. , Perez, R. Existence of Limit Cycles in the Repressilator equations. Intl. J. Bif. Chaos, Vol 19, No. 12 pp. 4097-4106 (2009).

[2] Buse, O., Perez, R., Kuznetsov,A. Dynamical properties of the repressilator model. Phys. Rev. E. Vol 81, 066206 (2010).

[3] Silvia De Monte, Francesco dOvidio, Sune Dan and Preben Graae Srensen, Dynamical quorum sensing: Population density encoded in cellular dynamics, PNAS vol. 104 (47) pp. 18377 (2007).

[4] Paula, Ana, Dias, S., Rodrigues, A. Hopf Bifurcation with $S_{N}$-symmetry, Nonlinearity, Vol 22 (2009) pp. 627-666.

[5] David Eberly, Geometric Tools, http://www.geometrictools.com/

[6] Ellowitz, M, Leibler, S. A synthetic oscillatory network of transcription regulators. Nature, Vol 403, pp. 335-338. (2000).

[7] Golubitsky, M., Stewart, I., Schaeffer, D. Singularities and Groups in Bifurcation Theory, Vol II, Appl. Math. Sci. 69, Springer, NewYork.

[8] T. Gedeon and K. Mischaikow, Structure of the global attractor of cyclic feedback systems J.Dynamics Di. Eq. 7 (1995), 141-190.

[9] T. Gedeon, Cyclic feedback systems, Memoirs of AMS 134 (637) (1998).

[10] Gonze, D. Modeling the effect of cell division on genetic oscillators. J. Theor. Biol., Vol 325. pp. 22-33 (2013).

[11] Koseska, A., Ullner, E. Volkov, E., Garcia-Ojalvo, J., Cooperative differentiation through clustering in Multicellular populations, J. Theor. Bio., vol 263, pp. 189-202 (2010).

[12] Levine, J., Lin, Y., Elowitz, M. Functional Roles of Pulsing in Genetic Circuits. Science, Vol 342, pp. 1193-1200 (2013).

[13] J. Mallet-Paret and H. Smith, The Poincare-Bendixson theorem for monotone cyclic feedback systems, J. Dynamics and Diff. Equations, 2 (1990), 367-421. 
[14] Muller, S., Hofbauer, J., Endler, L, Flamm, C., Widder, S., Schuster, P. A generalized model of the represillator. J. Math. Biol. Vol 53, pp. 905-937 (2006).

[15] Nandagopal, N., Elowitz, M. Synthetic Biology: Integrated Gene Circuits. Science, Vol 333, pp. 1244-1248 (2011).

[16] Jordi Garcia-Ojalvo, Michael B.Ellowitz and Steven H.Strogatz. Modeling a synthetic multicellular clock: repressilators coupled by quorum sensing. PNAS. Vol.101. No.30, 10955-10960 (2004).

[17] Pecora, L., Carroll, T., Master Stability Functions for Synchronized Coupled Systems. Phys. Rev. Letters, vol 80, no. 10, pp. 2109-2112 (1998).

[18] Potapov, I., Volkov, E., Kuznetsov, A., Dynamics of coupled repressilators: the role of mRNA kinetics and transcripton cooperativity. Phys. Rev. E, Vol 83, 031901 (2011).

[19] Potapov, I, Zhurov, B.I., Volkov Quorum sensing generated multistability and chaos in a synthetic genetic oscialltor. Chaos, Vol 22, 023117 (2012).

[20] Stewart, I., Symmetry methods in collisionless many-body problems. J. Nonlinear Sci., Vol 6, pp 543-563 (1996).

[21] Strelkowa, N., Barahona, M. Transient dynamics around unstable periodic orbits in the generalized repressilator model. Chaos, Vol 21, 023104 (2011).

[22] Thron, C.D., The secant condition for instability in biochemical feedback control: Parts I and II. Bull. Math. Biol. Vol 53, pp. 383-424 (1991).

[23] Tyson, J.J. and Othmer H.G., The dynamics of feedback control circuits in biochemical pathways. Progress in Theoretical Biology, Rosen R. and Snell F.M., ed. New York: Academic Press, Vol 5. (1978).

[24] Annette F. Taylor, Mark R. Tinsley, Fang Wang, Zhaoyang Huang and Kenneth Showalter, Dynamical quorum sensing and synchronization in large populations of chemical oscillators, Science, vol. 323 (614) pp. 614-619 (2009).

[25] Ullner, E., Zaikin, A., Volkov, E., Garcia-Ojalvo, J., Multistability and clustering in a population of synthetic genetic oscillators via phase-repulsive cell-to-cel communication. Phys. Rev. Letters, vol. 99, pp. 148103 (2007)

[26] Ullner, E., Koseska, A., Kurths, J., Volkov, E., Kantz, H., Garcia-Ojalvo, J., Multistability of syntheic genetic networks and repressive cell-to-cell communication, Phys. Rev. E., vol. 78, pp. 031904 (2008).

[27] Yang, Y., Kuznetsov, A., Characterization and merger of oscillatory mechanisms in an artificial genetic regulatory network, Chaos, vol 19, pp. 033115 (2009). 
[28] Zhou, T, Zhang, J., Yuan, Z., Chen, L. Synchronization of genetic oscillators. Chaos, Vol 18 , pp. 037126 (2008). 